\title{
Cytochrome $c$-Induced Increase of Motionally Restricted Lipid in Reconstituted Cytochrome $c$ Oxidase Membranes, Revealed by Spin-Label ESR Spectroscopy
}

\author{
Jörg H. Kleinschmidt, ${ }^{\ddagger}, \|$ Gary L. Powell,$^{\S}$ and Derek Marsh*,* \\ Max-Planck-Institut für biophysikalische Chemie, Abteilung Spektroskopie, Am Fassberg, D-37077 Göttingen, Germany, and \\ Department of Biological Sciences, Clemson University, Clemson, South Carolina 29634-1903
}

\begin{abstract}
Cytochrome $c$ oxidase isolated from beef heart mitochondria was reconstituted in bilayer membranes of the anionic lipid dimyristoylphosphatidylglycerol (DMPG) with varying enzyme/DMPG ratio. Lipid-protein interactions in the reconstituted membrane complexes were studied in the presence and absence of saturating amounts of bound cytochrome $c$, by both chemical binding assays and spinlabel ESR spectroscopy. The ESR spectra from a phosphatidylglycerol probe spin-labeled on C-14 of the $s n-2$ chain revealed two distinct lipid populations differing in their rotational mobility. The stoichiometry of lipids that were restricted in their rotational motion by direct interaction with the integral protein was 50-60 lipids/cytochrome $c$ oxidase monomer, in the absence of cytochrome $c$, independent of the total lipid/protein ratio. Cytochrome $c$ alone did not induce a motionally restricted population in the lipid ESR spectra, when bound to bilayers of negatively charged DMPG alone, in the fluid phase (at $36{ }^{\circ} \mathrm{C}$ ). However, the motionally restricted lipid population associated with reconstituted cytochrome $c$ oxidase/ DMPG membranes increased on binding cytochrome $c$, indicating structural/dynamic changes taking place in the membrane. Depending on the DMPG/cytochrome $c$ oxidase ratio, apparent stoichiometries of up to 115 motionally restricted lipid molecules/cytochrome $c$ oxidase monomer were found, when saturating amounts of cytochrome $c$ were bound. Under these conditions, cytochrome $c$ binds to $\sim 9$ negatively charged DMPG molecules, independent of the cytochrome $c$ oxidase content in the reconstituted system. A likely explanation for these results is that the surface binding of cytochrome $c$ propagates the motional restriction of the lipid chains beyond the first boundary shell of cytochrome $c$ oxidase, possibly creating microscopic in-plane domains.
\end{abstract}

In contrast to studies on the lipid interactions with integral proteins (e.g., ref 1 ), or on the binding of peripheral proteins to lipid membranes (e.g., ref 2), specific studies of the mutual effects of integral and peripheral membrane proteins on the membrane lipids are comparatively rare. Examples of the latter are spin-label ESR studies on myelin proteolipid and myelin basic protein reconstituted with negatively charged lipids (3), and on the binding of melittin (as a model for the regulatory protein phospholamban) to sarcoplasmic reticulum membranes containing the $\mathrm{Ca}^{2+}$-ATPase pump protein (4). Such studies are directly relevant to membrane structure, and to function, in the case of specific peripheral-integral protein couples.

Cytochrome $c$ oxidase is the terminal member of the mitochondrial electron transport chain (for reviews, see refs 5 , 6). The eukaryotic enzyme is a large integral membrane protein composed of 13 subunits, the structure of which has recently been determined by X-ray crystallography (7).

\footnotetext{
* Author to whom correspondence should be addressed. Telephone: +49 551201 1285. Fax: +49 551201 1501. E-mail: dmarsh@gwdg.de.

$\doteqdot$ Max-Planck-Institut für biophysikalische Chemie.

$\S$ Clemson University.

" Present address: University of Virginia HSC, Department of Molecular Physiology and Biological Physics, Charlottesville, VA 22906-0011.
}

Lipid-protein interactions with cytochrome $c$ oxidase have been characterized previously by spin-label ESR spectroscopy $(8-10)$. The electron-donating substrate of the oxidase complex, cytochrome $c$, the structure of which is also known (11), is a highly basic peripheral protein that binds to the surface of negatively charged lipid membranes $(12,13)$.

In the present work, we have concentrated on lipid-protein interactions for the cytochrome $c /$ cytochrome $c$ oxidase redox partners, reconstituted in bilayer membranes of the negatively charged phospholipid, dimyristoyl phosphatidylglycerol. Lipid-protein interactions of cytochrome $c$ oxidase alone in the latter lipid are found to parallel those found previously for this integral protein reconstituted in zwitterionic phosphatidylcholine (9). Binding of cytochrome $c$ increases, in a synergistic manner, these interactions of the negatively charged lipid with the integral protein, in a way that is quite unlike that of the interaction of cytochrome $c$ with membranes composed of negatively charged lipids alone. This is in contrast to the results of previous studies involving the myelin basic protein and proteolipid protein, where interactions were either merely additive, or ones of steric exclusion (3). In the cytochrome $c /$ cytochrome $c$ oxidase case, the mutual protein-lipid interactions create microdomains in which the lipid chain mobility is markedly reduced relative to that in fluid lipid membranes. 


\section{MATERIALS AND METHODS}

Materials. DMPG ${ }^{1}$ was obtained from Avanti (Alabaster, $\mathrm{AL}$ ) and was found to be pure by thin-layer chromatography using a solvent system $\mathrm{CHCl}_{3} / \mathrm{CH}_{3} \mathrm{OH} / \mathrm{ammonia}(65 / 30 / 3 \mathrm{v} / \mathrm{v} /$ v). Phosphatidylglycerol spin-labeled with the nitroxyl group on the C-14 atom of the $s n-2$ chain (14-PGSL) was synthesized from the corresponding spin-labeled stearic acid (14-SASL) (14). Horse heart cytochrome $c$ was from Sigma (St. Louis, MO). Cytochrome $c$ oxidase was prepared from beef heart by the method of Yu et al. (15) with modifications (16). Substitution of the enzyme-associated lipid by DMPG was carried out in a manner similar to that for the substitution by DMPC (17). Detergent-solubilized cytochrome $c$ oxidase was treated with a 500-fold molar excess of DMPG in $0.2 \%$ sodium cholate/10 mM HEPES, $1 \mathrm{mM}$ EDTA, $\mathrm{pH}$ 7.4, for $1 \mathrm{~h}$ at room temperature. After cooling the solution on ice, the enzyme was precipitated by adding cold concentrated ammonium sulfate solution to a final concentration of $35 \%$. The supernatant was removed after centrifugation at $4{ }^{\circ} \mathrm{C}$, and the lipid incubation and $\left(\mathrm{NH}_{4}\right)_{2} \mathrm{SO}_{4}$-precipitation steps were repeated a second time. Subsequently, the lipidsubstituted cytochrome $c$ oxidase was taken up in buffer containing $1 \%$ sodium cholate and centrifuged at $66000 \mathrm{~g}(4$ $\left.{ }^{\circ} \mathrm{C}\right)$. The solubilized preparation was assayed for protein and lipid content using protein and phosphate assays according to Lowry et al. (18) and Rouser et al. (19), respectively. The cytochrome $c$ oxidase-containing solution was then divided into several aliquots, and different amounts of DMPG in $0.2 \%$ cholate were added to each aliquot to give different molar ratios of DMPG to cytochrome $c$ oxidase. For cholate removal, samples were dialyzed against $10 \mathrm{mM}$ HEPES, $1 \mathrm{mM}$ EDTA, and $1 \mathrm{M} \mathrm{KCl}, \mathrm{pH}$ 7.4, at 4 ${ }^{\circ} \mathrm{C}$. The dialysis buffer was changed after 8 to $12 \mathrm{~h}$ for a total of three times. After dialysis, samples were washed and centrifuged two times in $10 \mathrm{mM}$ HEPES, $1 \mathrm{mM}$ EDTA, pH 7.4 buffer, without $\mathrm{KCl}$. Reconstituted samples were found to be homogeneous on continuous sucrose density gradients. Cytochrome $c$ oxidase activity after reconstitution was measured according to Yonetani (20). The activity of the samples reconstituted in DMPG was found to be $A=$ $5100 \mathrm{~min}^{-1}$, i.e., approximately half of the cytochrome $c$ oxidase activity in detergent before the reconstitution $(A=$ $11000 \mathrm{~min}^{-1}$ ). Activity after reconstitution in DMPG was therefore roughly comparable to the activity after reconstitution with other phospholipids (cf. ref 15).

ESR Sample Preparation and Sample Analysis. After dialysis, each sample containing DMPG and cytochrome $c$ oxidase at a given ratio was spin-labeled using a solution of 14-PGSL in ethanol, which was added such that the total amount of ethanol did not exceed $1 \%$ of the sample volume and the spin-label concentration was $1 \mathrm{~mol} \%$ of the total lipid. Each of the spin-labeled samples was divided into two aliquots. Membranes of the first aliquots were centrifuged, taken up in small amounts of buffer, transferred to ESR capillaries, and again centrifuged. ESR spectra were

\footnotetext{
${ }^{1}$ Abbreviations: BSA, bovine serum albumin; DMPC, 1,2-dimyristoyl-sn-glycero-3-phosphocholine; DMPG, 1,2-dimyristoyl-sn-glycero3-phosphoglycerol; 14-PGSL, 1-acyl-2-[14-(4,4-dimethyloxazolidine$\mathrm{N}$-oxyl)]stearoyl-sn-glycero-3-phosphoglycerol; EDTA, ethylenediaminetetraacetic acid; HEPES, $N$-(2-hydroxyethyl)piperazine- $N$ '-2-ethanesulfonic acid; ESR, electron spin resonance.
}

recorded after removal of the excess supernatant. A solution containing cytochrome $c(100 \mathrm{mg} / \mathrm{mL})$ was added to the second aliquot to give cytochrome $c / \mathrm{DMPG}$ ratios of $1 \mathrm{~mol} /$ mol. These samples were incubated for at least $20 \mathrm{~min}$ at room temperature, and subsequently the membrane complexes were separated by centrifugation. They were then washed two times with buffer to remove excess cytochrome $c$. The cytochrome $c /$ cytochrome $c$ oxidase/lipid complexes were transferred to ESR capillaries and packed by centrifugation. Control samples not containing cytochrome oxidase were prepared by dispersing DMPG plus 1 mol \% 14-PGSL, which had been dried down from chloroform solution, in buffer containing an excess of cytochrome $c$. Identical results and binding stoichiometry (9 DMPG/cytochrome $c$ ) were also obtained on adding excess cytochrome $c$ to DMPG dispersions of low ionic strength and incubating. All ESR samples were analyzed for their lipid and protein content after the spectra were recorded. Lipid/protein ratios of samples containing only DMPG and cytochrome $c$ oxidase were determined using Lowry protein assays with BSA as standard (18) and phosphate assays (19). The monomer molecular mass of cytochrome $c$ oxidase was taken to be $200 \mathrm{kDa}$. Cytochrome $c$ (molecular mass $12.4 \mathrm{kDa}$ ) was determined after dissolving the corresponding samples in buffer containing 5\% SDS. The absorbance differences at $550 \mathrm{~nm}$ of reduced and oxidized cytochrome $c$ were used and standardized on solutions of known concentrations in the same SDS-buffer. Reduction of the samples was performed with sodium dithionite. Treatment with dithionite did not affect the UV vis spectra of samples containing only cytochrome $c$ oxidase and DMPG. Samples were always kept on ice (or at $4{ }^{\circ} \mathrm{C}$ during centrifugation), except for the cytochrome $c$ incubation step and ESR data collection.

ESR Spectroscopy. ESR spectra were recorded at $36{ }^{\circ} \mathrm{C}$ on a $9 \mathrm{GHz}$ spectrometer (Century Line, E-12; Varian, Sunnyvale, CA) equipped with a $\mathrm{TE}_{102}$ rectangular cavity. Temperature was controlled within $\pm 0.1{ }^{\circ} \mathrm{C}$ by using nitrogen gas flow-temperature regulation and measured with a thin-wire thermocouple that was placed close to the sample at the top of the microwave cavity. Sealed sample capillaries were accommodated within standard quartz ESR tubes, which contained light silicone oil for thermal stability. A custom sample holder allowed positioning the sample in the cavity with an accuracy of $0.1 \mathrm{~mm}$. The spectrometer was interfaced to an IBM Personal Computer, using a Tecmar Labmaster A/D converter for digitizing and storing the ESR spectra. For improved sensitivity, $8-16$ spectral scans were accumulated, depending on the signal-to-noise ratio. The modulation frequency was $100 \mathrm{kHz}$ with a modulation amplitude of $1.25 \mathrm{G}$. A time constant of $0.25 \mathrm{~s}$ was chosen. Spectral subtractions were performed $(21)$ by using an interactive subtraction program written in $\mathrm{C}$. The digital subtractions allowed quantification of the two lipid populations found in the two-component ESR spectra.

\section{RESULTS}

Cytochrome c Binding to Reconstituted DMPG/Cytochrome c Oxidase Membranes. Cytochrome $c$ binds electrostatically to membranes containing negatively charged lipids (22). The stoichiometry of saturation binding to membranes composed of DMPG alone corresponds to 9 DMPG/cytochrome $c(12,13)$. Present work confirmed these 


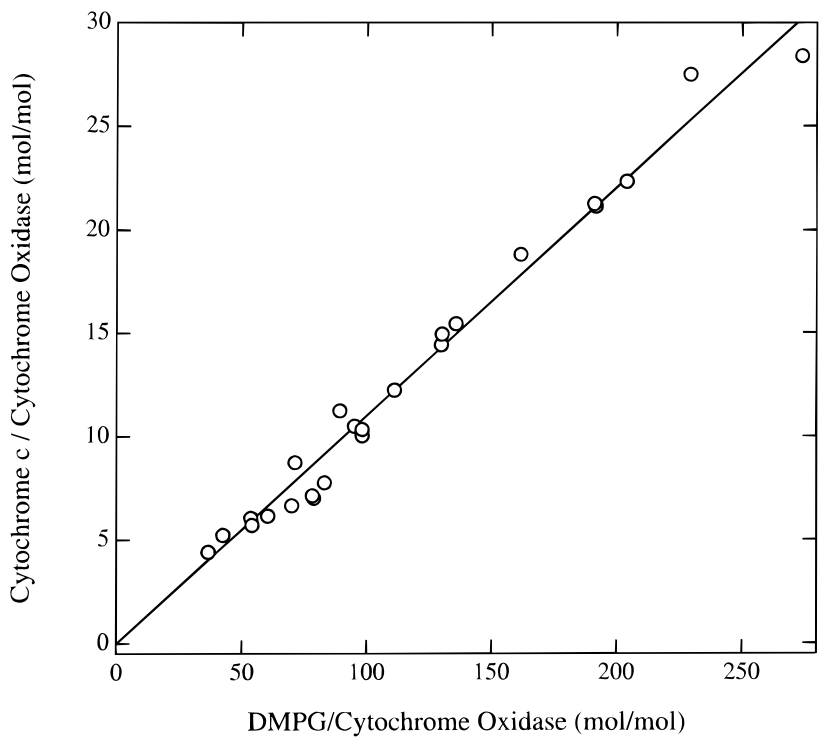

FIGURE 1: Binding of cytochrome $c$ to reconstituted cytochrome $c$ oxidase/DMPG membranes as a function of the DMPG/cytochrome $c$ oxidase molar ratio. Excess cytochrome $c$ was removed by washing twice with $10 \mathrm{mM}$ HEPES, $1 \mathrm{mM}$ EDTA, pH 7.4 buffer. Linear regression (continuous line) indicates that one cytochrome $c$ molecule binds to 9 DMPG molecules, independent of the amount of cytochrome $c$ oxidase reconstituted with DMPG.

findings. The saturation binding of cytochrome $c$ to reconstituted membranes of cytochrome $c$ oxidase and DMPG was determined as a function of the cytochrome $c$ oxidase/DMPG ratio by using a spectrophometric assay for cytochrome $c$, Lowry protein assay for cytochrome $c$ oxidase, and phosphate assay for DMPG. The cytochrome $c$ oxidase content of the reconstituted membranes was determined before addition of cytochrome $c$. All reconstituted cytochrome $c$ oxidase/ DMPG membranes of different lipid/protein ratios exhibited binding of cytochrome $c$ (Figure 1). The amount of bound cytochrome $c$, normalized to the cytochrome $c$ oxidase content of the membranes, is given in Figure 1 as a function of the DMPG/cytochrome $c$ oxidase ratio in the reconstituted samples. The relationship is approximately linear, and extrapolation to zero lipid content (i.e., cytochrome $c$ oxidase alone) indicates that relatively little cytochrome $c(-0.2 \pm$ $0.4 \mathrm{~mol} / \mathrm{mol}$ ) is bound to cytochrome $c$ oxidase under our experimental conditions (samples were washed with cytochrome $c$-free buffer after incubation with cytochrome $c$ ). The slope of the linear regression $(0.111 \pm 0.003 \mathrm{~mol} / \mathrm{mol})$ shows that about $9 \mathrm{~mol}$ of DMPG are bound by $1 \mathrm{~mol}$ of cytochrome $c$, independent of the cytochrome $c$ oxidase content of the membrane, as the lipid content of the membranes increases. This is the same as the stoichiometry of binding to DMPG membranes not containing cytochrome oxidase (see above).

14-PGSL ESR Spectra. The ESR spectra of the C-14 atom position phosphatidylglycerol spin label in (A) bilayers of DMPG alone to which a saturating amount of cytochrome $c$ is bound, (B) reconstituted cytochrome oxidase/DMPG membranes at a lipid/protein ratio of $160 \mathrm{~mol} / \mathrm{mol}$, and (C) the same cytochrome oxidase/DMPG membranes to which cytochrome $c$ is bound at saturation, are given in Figure 2. Spectra were recorded with a sample temperature of $36^{\circ} \mathrm{C}$, which is well above the chain melting transition of DMPG bilayer membranes (ca. $23{ }^{\circ} \mathrm{C}$ ). Saturation binding of

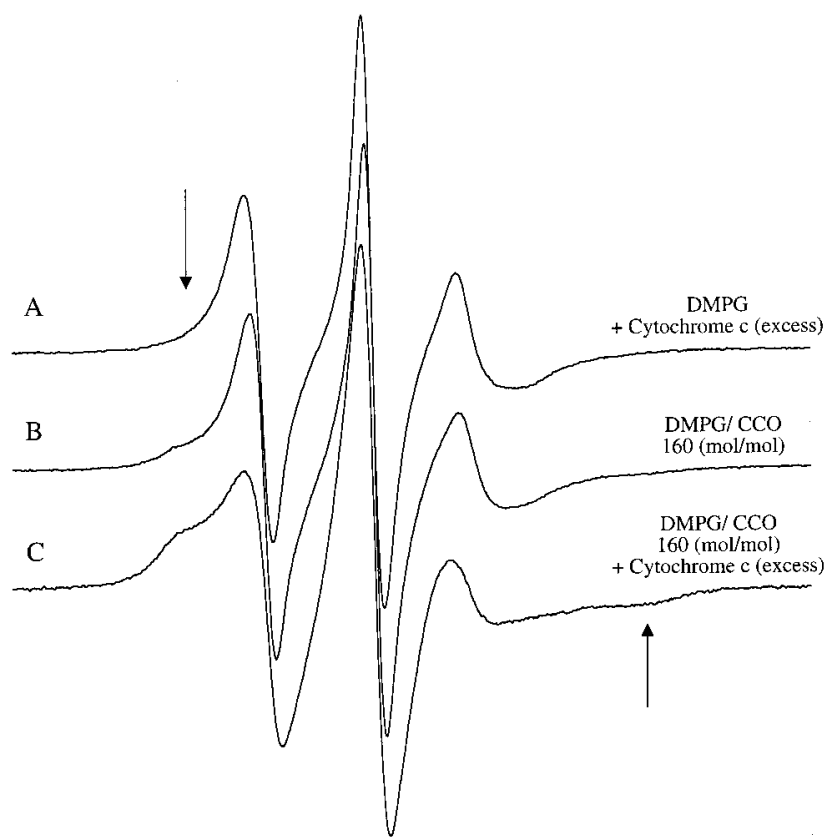

FIGURE 2: Effect of binding cytochrome $c$ on the motionally restricted lipid component in the ESR spectra of the 14-PGSL spin probe ( $1 \mathrm{~mol} \%$ of total lipid) in cytochrome $c$ oxidase/DMPG reconstituted membranes. ESR spectra from (A) DMPG and cytochrome $c$ alone, (B) reconstituted samples of DMPG and cytochrome $c$ oxidase at a ratio of $160 \mathrm{~mol} / \mathrm{mol}$, and (C) reconstituted cytochrome $c$ oxidase/DMPG $(1: 160 \mathrm{~mol} / \mathrm{mol}) \mathrm{mem}-$ branes with a saturating amount of cytochrome $c$ bound. The spectral component from motionally restricted lipids is indicated by the arrows. Spectral scan width: $100 \mathrm{G}, T=36{ }^{\circ} \mathrm{C}$.

cytochrome $c$ to the surface of DMPG bilayer membranes produces only a very limited decrease in mobility at the 14-C atom position of the lipid chains (cf. ref 12). The spectrum of 14-PGSL in Figure 2A is only slightly broader and more anisotropic than that of this label in bilayers of DMPG alone, at the same temperature. In contrast, the spectrum from 14PGSL in reconstituted DMPG/cytochrome oxidase membranes contains two distinct spin-label components at $36{ }^{\circ} \mathrm{C}$ (see Figure 2B). One of these components is similar to that of the spin label in DMPG bilayer membranes alone (or with cytochrome $c$ bound; Figure 2A). The other component corresponds to a population of lipids with a much reduced mobility that are interacting directly with the integral cytochrome oxidase enzyme. Qualitatively similar results have been obtained previously for cytochrome oxidase reconstituted in zwitterionic lipids such as dimyristoyl phosphatidylcholine (cf. refs 8, 9,23). Most interestingly, incubation of reconstituted samples of DMPG/cytochrome oxidase with excess cytochrome $c$ resulted in spectra in which the motionally restricted spin-label component (in the outer wings) was greatly increased (Figure 2C) compared with samples lacking cytochrome $c$, at the same temperature (cf. Figure 2B). This observation of a cytochrome $c$-induced increase in the population of motionally restricted lipid spin labels in DMPG/cytochrome $c$ oxidase membranes, although cytochrome $c$ binding to DMPG alone does not induce a second component in the outer wings of the spectra (Figure 2A), lead us to examine this effect at various ratios of DMPG/cytochrome $c$ oxidase. ESR spectra of 14-PGSL in samples of different DMPG/cytochrome $c$ oxidase ratios, both in the absence and in the presence of saturating amounts 
A
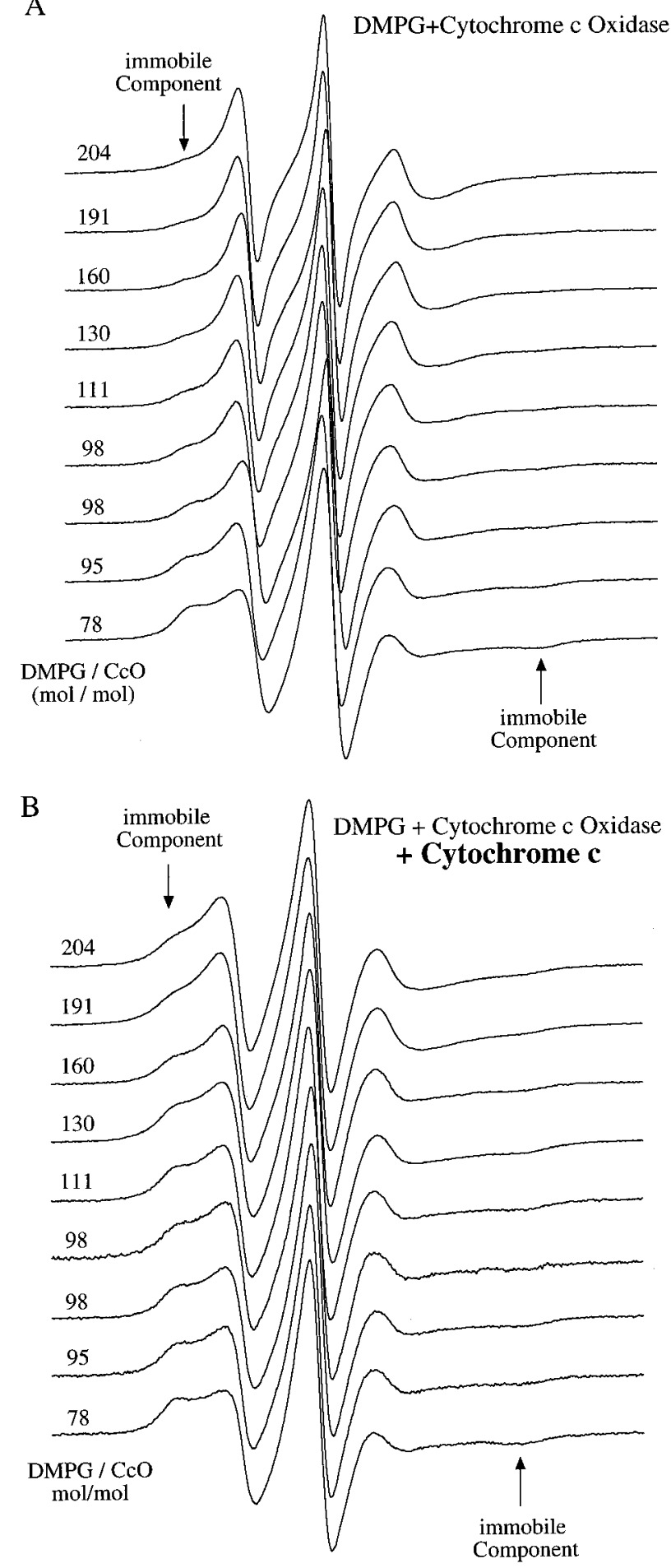

FIGURE 3: Effect of binding cytochrome $c$ on the ESR spectra of 14-PGSL $(1 \mathrm{~mol} \%)$ in DMPG/cytochrome $c$ oxidase membranes at various lipid/cytochrome $c$ oxidase ratios. Panel A: ESR spectra from reconstituted DMPG/cytochrome $c$ oxidase membranes alone, at the lipid/cytochrome $c$ oxidase molar ratios indicated. Panel B: ESR spectra from reconstituted membranes as in panel A, but with saturating amounts of cytochrome $c$ bound. Samples were in 10 mM HEPES, $1 \mathrm{mM}$ EDTA, pH 7.4 buffer. Spectral scan width $100 \mathrm{G}, T=36{ }^{\circ} \mathrm{C}$.

of cytochrome $c$, are shown in Figure 3. A large increase in the motionally restricted spin-label population was observed at all DMPG/cytochrome $c$ oxidase ratios investigated. To quantify this increase, spectral subtractions and integrations (cf. ref 21) were used to determine the relative populations of motionally restricted lipid in reconstituted membranes both with and without bound cytochrome $c$.

Quantitative Analysis of Motionally Restricted Lipid. For samples without cytochrome $c$, the ratio of the integrated intensities of the two resolved lipid spin-label components (fluid spin label/motionally restricted spin label) from DMPG/cytochrome $c$ oxidase complexes was plotted against the total lipid/protein ratio determined using standard lipid and protein assays (data not shown). A linear dependence was obtained $(r=0.98)$, as expected from the conventional equation for equilibrium lipid-protein association $(24,25)$ :

$$
n_{\mathrm{f}} / n_{\mathrm{b}}=n_{\mathrm{t}} /\left(N_{\mathrm{b}} K_{\mathrm{r}}\right)-1 / K_{\mathrm{r}}
$$

where $n_{\mathrm{f}} / n_{\mathrm{b}}$ is the ratio of fluid to motionally restricted populations of 14-PGSL, and $n_{\mathrm{t}}$ is the DMPG/cytochrome $c$ oxidase mole ratio. From the linear regression parameters, a value of $N_{\mathrm{b}} \approx 55-60$ motionally restricted lipids is found per cytochrome $c$ oxidase monomer, with a relative association constant for 14-PGSL of $K_{\mathrm{r}} \approx 1$. This result is in good agreement with studies on cytochrome $c$ oxidase reconstituted in lipid bilayers of zwitterionic dimyristoyl phosphatidylcholine with spin-labeled phosphatidylcholine $(9,10)$. Cytochrome $c$ oxidase therefore does not exhibit a measurable specificity for spin-labeled relative to unlabeled phospholipid, when both the host lipid and the spin-labeled lipid have the same headgroup. Characterization of cytochrome oxidase reconstituted in DMPC by freeze fracture revealed a random dispersion of proteins in fluid-phase membranes $(9,26)$. The similar stoichiometries of motionally restricted (i.e., perimeter) lipids in DMPC and DMPG therefore indicate that cytochrome oxidase is similarly dispersed in DMPG.

To compare the results obtained with cytochrome $c$ oxidase/DMPG membranes with those from similar samples with bound cytochrome $c$, the relative population of motionally restricted lipid, normalized to the content of cytochrome $c$ oxidase integral protein in the membranes, is plotted as a function of the total lipid/cytochrome $c$ oxidase ratio in Figure 4. The results of spectral subtractions, for samples containing cytochrome $c$ oxidase and DMPG alone, show that the number of restricted lipids remains close to 55 lipids per cytochrome $c$ oxidase, independent of the total lipid/cytochrome $c$ oxidase ratio. However, reconstituted membranes saturated with cytochrome $c$ contain amounts of motionally restricted lipids that increase progressively with the lipid/cytochrome $c$ oxidase ratio, at least in the region of DMPG/cytochrome $c$ oxidase ratios investigated here. Therefore, there is a greater population of motionally restricted lipid, the higher is the cytochrome c/cytochrome $c$ oxidase ratio (cf. Figure 1). Up to 115 motionally restricted phospholipids were found in the double reconstituted system at a mole ratio of DMPG/cytochrome $c$ oxidase of 200. More than half of the total spin-labeled lipid population is motionally restricted throughout the range of DMPG/ cytochrome $c$ oxidase ratios $\left(n_{\mathrm{t}}\right)$ studied. Also this fraction changes only slowly with increasing lipid/protein ratio, remaining at $70-75 \%$ up to $n_{\mathrm{t}}=130 \mathrm{~mol} / \mathrm{mol}$, and decreasing to ca. $60 \%$ over the range of $n_{\mathrm{t}}=130-200 \mathrm{~mol} /$ mol.

A topic of considerable interest is the extent to which the additional population of lipids that are restricted by the 


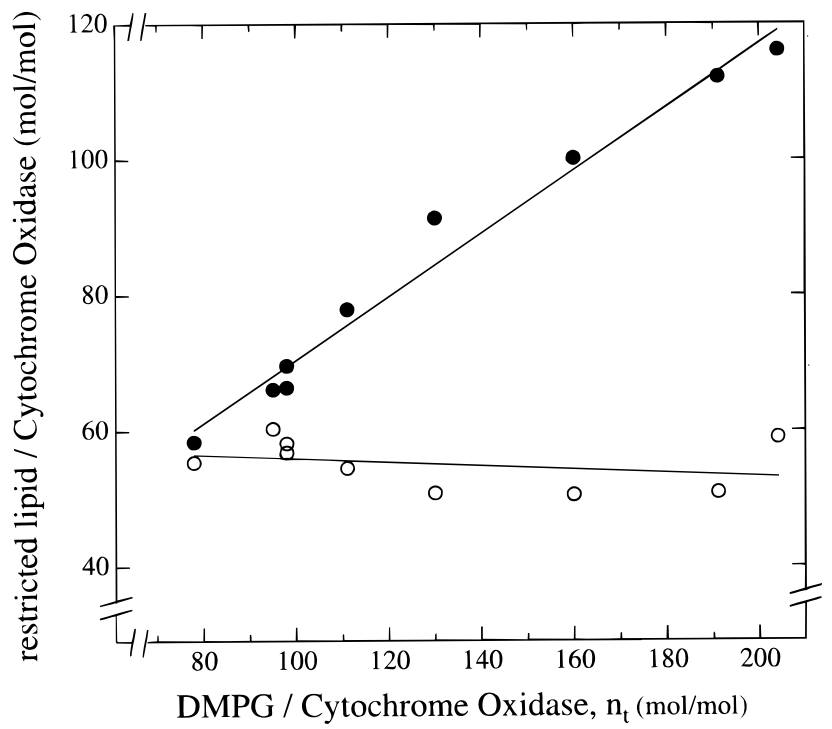

FIGURE 4: Motionally restricted lipid population in reconstituted complexes of cytochrome $c$ oxidase with DMPG, in the absence (open circles) and in the presence (filled circles) of bound cytochrome $c$, as a function of the lipid/cytochrome $c$ oxidase ratio, $n_{\mathrm{t}}$. Stoichiometries, $N_{\mathrm{b}} \equiv f n_{\mathrm{t}}$, of motionally restricted lipid per cytochrome $c$ oxidase monomer were calculated assuming equivalence between 14-PGSL and DMPG (i.e., $K_{\mathrm{r}} \approx 1$ in the absence of cytochrome $c$ ).

Table 1: Outer Hyperfine Splitting Constants, $A_{\max }$, of the Difference Spectra Corresponding to the Motionally Restricted Spin-labeled Lipid (14-PGSL) Population in Cytochrome Oxidase/ DMPG Membranes of Different Lipid/Protein Ratios, in the Presence and Absence of Saturating Amounts of Cytochrome $c$

\begin{tabular}{cll}
\hline \multirow{2}{*}{$\begin{array}{c}\text { DMPG/cyt oxidase } \\
(\mathrm{mol} / \mathrm{mol})\end{array}$} & \multicolumn{2}{c}{$A_{\max }$ (gauss) } \\
\cline { 2 - 3 } 78 & 29.4 & + cyt $c$ \\
95 & 29.3 & 28.9 \\
98 & 29.4 & 29.0 \\
111 & 29.2 & 29.1 \\
130 & 29.4 & 29.5 \\
160 & 29.7 & 28.6 \\
191 & 29.0 & 28.5 \\
mean & $29.3 \pm 0.2$ & 29.2 \\
\hline
\end{tabular}

synergistic action of binding cytochrome $c$ are reduced in their mobility. The difference spectra that correspond to the total motionally restricted spin-labeled lipid population in the presence of cytochrome $c$ still approximate those of a single component that lies in the slow-motion regime of conventional spin-label ESR. The outer hyperfine splittings, $2 A_{\max }$, of these difference spectra were used to characterize the mobility of the motionally restricted lipids. The values of $A_{\max }$ given in Table 1 show, as expected (cf. ref 9), that there is little dependence on the DMPG/cytochrome oxidase ratio and that these values are not changed appreciably by the binding of cytochrome $c$. Thus, both the first-shell lipids associated with cytochrome oxidase, and the additional lipids that are restricted by the synergistic effect of binding cytochrome $c$, have rotational motions that lie in the slowmotional regime, in which the ESR spectra are not very sensitive to differences in mobility (e.g., ref 27). This does not imply that the degree of motional restriction of the lipids that are synergistically affected by the binding of cytochrome $c$ is identical with that of the first shell of lipids surrounding cytochrome oxidase. It is simply that the mobility of both lipid populations lies beyond the time scale of optimum sensitivity of conventional spin-label ESR spectroscopy.

\section{DISCUSSION}

Cytochrome c Binding to Reconstituted DMPG/Cytochrome c Oxidase Membranes. The binding of cytochrome $c$ to reconstituted membranes of DMPG and cytochrome $c$ oxidase is dependent on the content of DMPG lipid only, and to a good approximation does not depend on the presence of cytochrome $c$ oxidase. Extrapolation to zero lipid content of the linear fit in Figure 1 also shows that cytochrome $c$ oxidase does not bind cytochrome $c$ appreciably under our experimental conditions, certainly not more than one cytochrome $c$ per cytochrome oxidase. It should be noted that all samples containing cytochrome $c$ were washed twice with cytochrome $c$-free buffer prior to the binding assays. Interestingly, the data indicate that even the lipid in direct contact with the hydrophobic surface of cytochrome $c$ oxidase is still available for binding cytochrome $c$ because the binding stoichiometry is the same as that for saturation binding to DMPG alone, which corresponds to complete surface coverage (cf. ref 22). This suggests that binding of cytochrome $c$ might cause cross-bridging of the cytochrome $c$ oxidase molecules at the lower lipid-to-enzyme ratios.

It is interesting to compare these findings with the results obtained previously on the double-reconstituted system of the peripheral basic protein and the integral proteolipid protein of nerve myelin with DMPG (3). In experiments similar to those reported here, it was found that the DMPG lipid in direct contact with the myelin proteolipid protein is not available for binding the myelin basic protein, which is in direct contrast to the present results for the cytochrome $c /$ cytochrome $c$ oxidase/DMPG double-reconstituted system. The data for the myelin proteins was interpreted in terms of a mutual steric exclusion model in which the boundary layer of lipid surrounding the proteolipid protein is excluded from interaction with the basic protein, and the latter binds only to the bulk (i.e., nonboundary) lipid surface. Both proteolipid and myelin basic proteins exhibit a strong selectivity for negatively charged lipids $(28,29)$. Our finding that cytochrome $c$ binds even to the lipids adjacent to cytochrome $c$ oxidase (i.e., no steric exclusion) suggests a rather strong electrostatic binding of cytochrome $c$ to negatively charged lipids such as DMPG. This interaction is apparently stronger than the electrostatic interaction between DMPG and the integral cytochrome $c$ oxidase protein, even though cytochrome $c$ oxidase does show selectivity for certain negatively charged lipids over neutral lipids $(10,16,23)$. In this context, it is interesting that model membranes reconstituted from cytochrome $c$ oxidase and zwitterionic DMPC do not exhibit any significant net electrostatic potential (Kleinschmidt et al., unpublished results) and that cytochrome $c$ oxidase transiently binds positively charged cytochrome $c$ in its reduced form during the enzymatic oxidation process, which would not occur if there were a steric exclusion similar to that found for myelin basic/proteolipid protein recombinants with DMPG.

Motional Restriction of Lipids. The existence of motionally restricted lipid in bilayer membranes containing a reconstituted integral protein has been interpreted, from its stoichiometry, as a boundary layer, or first shell, of lipid 
surrounding the hydrophobic intramembranous surface of the protein $(8,30)$. The stoichiometry of lipids motionally restricted by cytochrome $c$ oxidase reconstituted in DMPG is found to be the same as that for reconstitutions in zwitterionic phosphatidylcholine lipids (9), further verifying that these lipids correspond operationally to a first shell around the integral protein. Comparisons with the recent high-resolution structure of the 13-subunit cytochrome $c$ oxidase from beef heart ( 7 ) further suggest that this functional first shell of lipids also corresponds to the actual perimeter lipids surrounding the intramembranous section of the protein (e.g., ref 1). Binding of saturating amounts of cytochrome $c$ increases the motionally restricted lipid population in membranes of cytochrome $c$ oxidase reconstituted with DMPG. Moreover, the increase is (at least at the lower lipid/ enzyme ratios) proportional to the DMPG/cytochrome $c$ oxidase ratio. It is also remarkable that the increase in motionally restricted lipid population is rather large, with the amount of restricted lipid being doubled at a DMPG/ cytochrome $c$ oxidase ratio of $\sim 180 \mathrm{~mol} / \mathrm{mol}$. A single cytochrome $c$ oxidase molecule was found to have a boundary population of about 55 DMPG molecules in the absence of cytochrome $c$. When there are roughly three times as many lipids present as are required to form the boundary layer, addition of cytochrome $c$ causes almost twothirds of these to be motionally restricted.

One way to interpret the increase in motional restriction is as a propagation of the immobilization effect of lipids in the presence of cytochrome $c$, extending the chain restriction from the first lipid shell around cytochrome $c$ oxidase to lipids in the second and possibly even third shell around the protein. Cytochrome $c$ oxidase has been found to have a considerably invaginated intramembranous surface (7). However, it is unlikely that conformational changes in the enzyme would result in an increase of its hydrophobic surface large enough to account for such a dramatic increase in population as that observed at high lipid/enzyme ratios. The likelihood is therefore that the binding of cytochrome $c$ to reconstituted DMPG membranes causes a propagation in the motional restriction of the lipid chains that are directly in contact with the intramembranous surface of cytochrome $c$ oxidase. The mechanism for this is not known with certainty, but there exist two general possibilities: either by a direct or by an indirect interaction with cytochrome $c$. The indirect mechanism would involve aggregation of cytochrome $c$ oxidase by interaction with cytochrome $c$. An increase in population of motionally restricted chains could then arise from trapping of lipids within the cytochrome $c$ oxidase aggregates. This mechanism again is less likely because of the relatively large number of lipids that would have to be accommodated at high lipid/protein ratios (up to four lipid shells between adjacent cytochrome oxidase molecules), which per force must tend to disperse the cytochrome $c$ oxidase aggregates. Additionally, the stoichiometry of cytochrome $c$ binding to cytochrome oxidase/DMPG membranes corresponds to complete coverage of the DMPG membrane surface implying that any notionally "trapped" lipids must be in contact with cytochrome $c$ and not segregated from it. On the other hand, a propagation of lipid immobilization could take place by direct interaction with cytochrome $c$, which can bind to the lipid surface even close to cytochrome $c$ oxidase, as is indicated by Figure 1. We found that cytochrome $c$ binds to about 9 DMPG molecules and it could therefore form a surface-bridge between lipids in the first boundary layer of cytochrome $c$ oxidase and those in layers of higher order (next to the first boundary shell). Such a mechanism is possible because, even though binding of cytochrome $c$ alone does not create a specific population of motionally restricted lipids, it does induce a generalized increase in motional restriction of the entire population of lipid chains, at saturation binding (see Figure 2A, and refs 12, 13). Therefore a synergistic effect of surface binding of cytochrome $c$ on the motional restriction of the lipid chains by the intramembranous section of cytochrome $c$ oxidase may lead to motional restriction of subsequent lipid shells.

Again, it is interesting to compare the data reported here for the cytochrome $c /$ cytochrome $c$ oxidase complex with earlier studies on myelin proteolipid/basic protein recombinant DMPG membranes (3). It was found that around 10 molecules of DMPG were motionally restricted per proteolipid protein molecule, in the absence of myelin basic protein (proteolipid protein $M_{\mathrm{r}} \approx 25 \mathrm{kDa}$, is much smaller than cytochrome $c$ oxidase, $M_{\mathrm{r}} \approx 200 \mathrm{kDa}$ ), consistent with earlier studies using a zwitterionic lipid environment (28). However, when the myelin basic protein was added, the stoichiometry of motionally restricted lipid decreased, which differs from the results reported here on cytochrome $c /$ cytochrome $c$ oxidase/DMPG. It was suggested that the myelin basic protein disturbs the interaction of lipids with the intramembranous portion of the proteolipid protein, possibly by means of the membrane-penetrant sections of former (cf. ref 29). Addition of cytochrome $c$ to reconstituted lipid-protein membranes, on the other hand, cannot result in such an effect because this peripheral protein was found not to penetrate the lipid bilayer (12). Therefore, the effects that a peripheral protein may cause on the interface between integral membrane proteins and the fluid membrane lipids could depend to a large extent on the ability of the peripheral protein to penetrate the lipid bilayer, and correspondingly could also depend on the functional properties of the integral proteinperipheral protein couples, viz., a structural role in the case of the proteolipid and basic proteins of myelin, and an enzymatic redox couple in the case of mitochondrial cytochrome $c$ and cytochrome $c$ oxidase.

\section{CONCLUSION}

The results of this study therefore indicate that binding of cytochrome $c$ to reconstituted membranes composed of negatively charged lipid and containing cytochrome $c$ oxidase can induce formation of microdomains in which the rotational mobility of the lipid chains is appreciably restricted relative to that in fluid lipid membranes. This is in contrast to structural interactions of the myelin proteolipid and basic proteins in double-reconstituted membranes (3), but qualitatively similar to the interactions of melittin with the CaATPase and associated lipids in sarcoplasmic reticulum membranes (4). The size and configuration of the lipidprotein domains in the cytochrome $c /$ cytochrome $c$ oxidase system is not known, but the effective lipid/protein stoichiometry can reach quite high values, up to $120: 1 \mathrm{~mol} / \mathrm{mol}$ at high total lipid/protein ratios. A possible functional significance for this lipid domain formation remains speculative, but it is clear that it might function to concentrate and channel the cytochrome $c$ substrate to the integral oxidase enzyme. 
The inner mitochondrial membrane is specifically enriched in the negatively charged lipid diphosphatidylglycerol (i.e., cardiolipin) (31), which might be even more effective in this respect than is monophosphatidylglycerol. Certainly, diphosphatidylglycerol is considerably enriched relative to monophosphatidylglycerol, in its selective association with cytochrome $c$ oxidase (10).

\section{ACKNOWLEDGMENT}

We thank Frau B. Angerstein for the preparation of phospholipid spin labels.

\section{REFERENCES}

1. Marsh, D., and Horváth, L. I. (1998) Biochim. Biophys. Acta (in press).

2. Sankaram, M. B., and Marsh, D. (1993) in New Comprehensive Biochemistry, Vol. 25. Protein-Lipid Interactions (Watts, A., Ed.) pp 127-162, Elsevier, Amsterdam, The Netherlands.

3. Sankaram, M. B., Brophy, P. J., and Marsh, D. (1991) Biochemistry 30, 5866-5873.

4. Mahaney, J. E., Kleinschmidt, J., Marsh, D., and Thomas, D. D. (1992) Biophys. J. 63, 1513-1522.

5. Musser, S. M., Stowell, M. H., and Chan, S I. (1995) Adv. Enzymol. Relat. Areas Mol. Biol. 71, 79-208.

6. Brzezinski, P. (1996) Biochemistry 35, 5612-5615.

7. Tsukihara, T., Aoyama, H., Yamashita, E., Tomizaki, T., Yamaguchi, H., Shinzawa-Itoh, K., Nakashima, R., Yaono, R., and Yoshikawa, S. (1996) Science 272, 1136-1144.

8. Jost, P. C., Griffith, O. H., Capaldi, R. A., and Vanderkooi, G. (1973) Proc. Natl. Acad. Sci. U.S.A. 70, 480-484.

9. Knowles, P. F., Watts, A., and Marsh, D. (1979) Biochemistry 18, 4480-4487.

10. Knowles, P. F., Watts, A., and Marsh, D. (1981) Biochemistry 20, 5888-5894.

11. Bushnell, G. W., Louie, G. V., and Brayer, G. D. (1990) J. Mol. Biol. 214, 585-595.

12. Görrisen, H., Marsh, D., Rietveld, A., and de Kruijff, B. (1986) Biochemistry 25, 2904-2910.
13. Sankaram, M. B., de Kruijff, B., and Marsh, D. (1989) Biochim. Biophys. Acta 986, 315-320.

14. Marsh, D., and Watts, A. (1982) in Lipid-Protein Interactions. Vol. 2 (Jost, P. C., and Griffith, O. H., Eds.) pp 53-126, Wiley-Interscience, New York.

15. Yu, C., Yu, L., and King, T. E. (1975) J. Biol. Chem. 250, $1383-1392$.

16. Cable, M. B., and Powell, G. L. (1980) Biochemistry 19, 5679-5686.

17. Watts, A., Marsh, D., and Knowles, P. F. (1978) Biochem. Biophys. Res. Commun. 81, 403-409.

18. Lowry, O. H., Rosebrough, N. J., Farr, A. L., and Randall, R. J. (1951) J. Biol. Chem. 193, 265-275.

19. Rouser, G., Fleischer, S., and Yamamoto, A. (1970) Lipids 5, 494-496.

20. Yonetani, T. (1966) Biochem. Prep. 11, 14-20.

21. Marsh, D. (1982) Techn. Life Sci.: Biochem. B4/II, B426/1B426/44.

22. Heimburg, T., and Marsh, D. (1995) Biophys. J. 68, 536546.

23. Powell, G. L., Knowles, P. F., and Marsh, D. (1987) Biochemistry 26, 8138-8145.

24. Brotherus, J. R., Griffith, O. H., Brotherus, M. O., Jost, P. C., Silvius, J. R., and Hokin, L. E. (1981) Biochemistry 20, 52615267.

25. Marsh, D. (1985) in Progress in Protein-Lipid Interactions, Vol. 1 (Watts, A., and de Pont, J. J. H. H. M., Eds.) pp 143172, Elsevier, Amsterdam, The Netherlands.

26. Fajer, P., Knowles, P. F., and Marsh, D. (1989) Biochemistry $25,5634-5643$.

27. Freed, J. H. (1976) in Spin Labeling. Theory and Applications (Berliner, L. J., Ed.) pp 53-132, Academic Press Inc., New York.

28. Brophy, P. J., Horváth, L. I., and Marsh, D. (1984) Biochemistry 23, 860-865.

29. Sankaram, M. B., Brophy, P. J., and Marsh, D. (1989) Biochemistry 28, 9685-9691.

30. Marsh, D. (1997) Eur. Biophys. J. 26, 203-208.

31. Daum, G. (1985) Biochim. Biophys. Acta 822, 1-42.

BI980235B 